\title{
Local Permutations of Products of Bell States and Entanglement Distillation
}

\author{
Jeroen Dehaene, ${ }^{\text {f }}$ Maarten Van den Nest, and Bart De Moor \\ Katholieke Universiteit Leuven, ESAT-SCD, Belgium \\ Frank Verstraete \\ Universiteit Gent, Department of Mathematical Physics and Astronomy, Belgium
}

(Dated: September 4, 2018)

\begin{abstract}
We present new algorithms for mixed-state multi-copy entanglement distillation for pairs of qubits. Our algorithms perform significantly better than the best known algorithms. Better algorithms can be derived that are tuned for specific initial states. The new algorithms are based on a characterization of the group of all locally realizable permutations of the $4^{n}$ possible tensor products of $n$ Bell states.
\end{abstract}

PACS numbers: 03.67.-a

\section{INTRODUCTION}

We study mixed-state multi-copy entanglement distillation protocols for pairs of qubits. We start from $n$ identical copies of a Bell diagonal state of 2 qubits and end up, after local operations and classical communication, with $m<n$ Bell diagonals (possibly statistically dependent) with higher joint fidelity than $m$ copies of the original Bell diagonal state. For non-Bell-diagonal initial states one can first perform $n$ separate optimal single-copy distillation protocols to make them Bell-diagonal [1]. Our protocol can be used in a recurrence scheme followed by the hashing protocol as in [2, 3. We propose a protocol with $n=4$ and $m=1$ that does significantly better than existing protocols. Our results can be used to find even better protocols for other values of $n$ and $m$ that are tuned for specific initial states.

We see three main reasons for studying entanglement distillation protocols. The first and most obvious reason is that entanglement distillation protocols are a means of obtaining states that are closer to maximally entangled pure states, as needed in typical applications like teleportation, from mixed states that can be reached by sending one qubit of an entangled pair through a realistic channel. A second reason to study distillation protocols is that asymptotic protocols yield a lower bound for "entanglement of distillation", an important measure of entanglement, that is in itself a lower bound for any sensible measure of entanglement [4]. In this context we also mention the upper-bounds on entanglement of distillation obtained in 5.5. A third reason is that multi-copy entanglement protocols can be considered as applications of entanglement, where more can be done in the presence of entangled pairs than without. We hope that studying these mechanisms will reveal some information on the important problem of how exactly the presence of en-

\footnotetext{
*Electronic address: Jeroen.Dehaene@esat.kuleuven.ac.be
}

${ }^{\dagger}$ Also at K.U.Leuven, ESAT-SCD tanglement enables one to do things that are impossible without.

Multi-copy mixed state entanglement distillation for qubit pairs was first studied in [2, 3]. An improved variant of the two-copy protocol in that paper was described in [6] under the title of quantum privacy amplification. These protocols as well as ours start from $n$ identical qubit pairs in a Bell-diagonal state, shared by Alice and Bob. A crucial ingredient of these protocols is a local unitary operation, performed by Bob and Alice on their $n$ qubits, that results globally in a permutation of the $4^{n}$ possible tensor products of Bell states. The key ingredient of this paper is a characterization, by means of a binary matrix group, of all possible local permutations of the products of Bell states. This enables a search for the best protocol within this setting. In section II we study local permutations of products of Bell states. In section III we discuss the new protocols. In section IV we discuss the combination of our protocols with a recurrence scheme and the hashing protocol and show the strength of our protocols by computer simulations.

\section{LOCAL PERMUTATIONS OF PRODUCTS OF BELL STATES}

In this section we study the class of local unitary operations that can be performed by Alice and Bob locally and result in a permutation of the $4^{n}$ (tensor) products of Bell states, where $n$ is the number of qubit pairs. These local permutations are the key ingredient of the new distillation protocols described in the next section.

We will code products of Bell states by binary vectors by assigning two-bit vectors to the Bell states as follows

$$
\begin{aligned}
& \left|\Phi^{+}\right\rangle=\frac{1}{\sqrt{2}}(|00\rangle+|11\rangle)=\left|B_{00}\right\rangle \\
& \left|\Psi^{+}\right\rangle=\frac{1}{\sqrt{2}}(|01\rangle+|10\rangle)=\left|B_{01}\right\rangle \\
& \left|\Phi^{-}\right\rangle=\frac{1}{\sqrt{2}}(|00\rangle-|11\rangle)=\left|B_{10}\right\rangle \\
& \left|\Psi^{-}\right\rangle=\frac{1}{\sqrt{2}}(|01\rangle-|10\rangle)=\left|B_{11}\right\rangle .
\end{aligned}
$$

A product of $n$ Bell states is then described by a 
$2 n$-bit vector, e.g. $\left|B_{001101}\right\rangle=\left|B_{00}\right\rangle\left|B_{11}\right\rangle\left|B_{01}\right\rangle=$ $\left|\Phi^{+}\right\rangle\left|\Psi^{-}\right\rangle\left|\Psi^{+}\right\rangle$.

We will also exploit a correspondence between Bell states and Pauli matrices

$$
\begin{aligned}
& \left|\Phi^{+}\right\rangle \rightarrow \frac{1}{\sqrt{2}} \sigma_{00}=\frac{1}{\sqrt{2}} \sigma_{0}=\frac{1}{\sqrt{2}}\left[\begin{array}{ll}
1 & 0 \\
0 & 1
\end{array}\right] \\
& \left|\Psi^{+}\right\rangle \rightarrow \frac{1}{\sqrt{2}} \sigma_{01}=\frac{1}{\sqrt{2}} \sigma_{x}=\frac{1}{\sqrt{2}}\left[\begin{array}{ll}
0 & 1 \\
1 & 0
\end{array}\right] \\
& \left|\Phi^{-}\right\rangle \rightarrow \frac{1}{\sqrt{2}} \sigma_{10}=\frac{1}{\sqrt{2}} \sigma_{z}=\frac{1}{\sqrt{2}}\left[\begin{array}{rr}
1 & 0 \\
0 & -1
\end{array}\right] \\
& \left|\Psi^{-}\right\rangle \rightarrow \frac{1}{\sqrt{2}} \sigma_{11}=\frac{1}{\sqrt{2}} \sigma_{y}=\frac{1}{\sqrt{2}}\left[\begin{array}{rr}
0 & -i \\
i & 0
\end{array}\right]
\end{aligned}
$$

A tensor product of $n$ Bell states is then described by a tensor product of Pauli matrices, e.g. $\left|\Phi^{+}\right\rangle\left|\Psi^{-}\right\rangle\left|\Psi^{+}\right\rangle \rightarrow$ $\frac{1}{\sqrt{8}} \sigma_{0} \otimes \sigma_{y} \otimes \sigma_{x}=\frac{1}{\sqrt{8}} \sigma_{00} \otimes \sigma_{11} \otimes \sigma_{01}$. We will also use longer vector subscripts to denote such tensor products, e.g.

$$
\sigma_{001101}=\sigma_{00} \otimes \sigma_{11} \otimes \sigma_{01} .
$$

In this representation of pure states of $2 n$ qubits as $2^{n} \times$ $2^{n}$-matrices $\tilde{\Psi}$, local unitary operations $|\psi\rangle \rightarrow\left(U_{A} \otimes\right.$ $\left.U_{B}\right)|\psi\rangle$, in which Alice acts on her $n$ qubits (jointly) with an operation $U_{A}$ and Bob on his with an operation $U_{B}$, are represented by

$$
\tilde{\Psi} \rightarrow U_{A} \tilde{\Psi} U_{B}^{T}
$$

We are now in a position to state the main result of this section:

Theorem 1 (i) If a local unitary operation (3) results in a permutation of the $4^{n}$ tensor products of $n$ Bell states, this permutation can be represented on the binary vector representations (1) as an affine operation

$$
\begin{array}{ll} 
& \phi: \mathbb{Z}_{2}^{2 n} \rightarrow \mathbb{Z}_{2}^{2 n}: x \rightarrow A x+b \\
\text { with } & A \in \mathbb{Z}_{2}^{2 n \times 2 n}, b \in \mathbb{Z}_{2}^{2 n} \\
\text { and } & A^{T} P A=P \\
\text { where } & P=\operatorname{diag}\left[\left[\begin{array}{ll}
0 & 1 \\
1 & 0
\end{array}\right], \ldots,\left[\begin{array}{ll}
0 & 1 \\
1 & 0
\end{array}\right]\right]
\end{array}
$$

(ii) Conversely, any such permutation can be realized by local unitary operations.

Note that all multiplication and addition should be done modulo 2. We call a matrix $A$ satisfying $A^{T} P A=P$ $P$-orthogonal. The affine and linear transformations considered are invertible and therefore amount to a permutation of $\mathbb{Z}_{2}^{2 n}$. In the sequel we sometimes directly refer to the linear transformations as permutations.

Proof: We first prove part (i). One can easily check that $\sigma_{v} \cdot \sigma_{w} \sim \sigma_{v+w}$, where $v$ and $w$ are binary vector indices as in (2), and the $\sim$-sign means equal up to a complex phase (in this case $1, i,-1$ or $-i$ ). Such a phase is irrelevant as the Pauli matrices here are matrix representations of pure state vectors.

Assume now that $U_{A}$ and $U_{B}$ indeed result in a permutation $\pi: \mathbb{Z}_{2}^{2 n} \rightarrow \mathbb{Z}_{2}^{2 n}$, then the null vector is mapped to some vector $v=\pi(0)$. Accordingly $U_{A} \sigma_{0} U_{B}^{T} \sim \sigma_{v}$. Since $\sigma_{0}$ is the identity matrix, we have $U_{B} \sim \sigma_{v} U_{A}^{*}$ where * denotes complex conjugation. If we want to represent $\pi$ by $x \rightarrow A x+b$ we clearly have to choose $b=v$. Note that (3) now reads $\tilde{\Psi} \rightarrow U_{A} \tilde{\Psi} U_{A}^{\dagger} \sigma_{b}$.

We now have to show that the permutation $\pi^{\prime}: x \rightarrow$ $\pi(x)+b$, which maps $\tilde{\Psi}$ to $U_{A} \tilde{\Psi} U_{A}^{\dagger}$, is a linear $P$ orthogonal map $\pi^{\prime}: x \rightarrow A x$. Linearity of binary maps means that sums are mapped to sums $\pi^{\prime}(v+w)=$ $\pi^{\prime}(v)+\pi^{\prime}(w)$. This is clearly true since $U_{A} \sigma_{v+w} U_{A}^{\dagger} \sim$ $U_{A} \sigma_{v} \sigma_{w} U_{A}^{\dagger}=U_{A} \sigma_{v} U_{A}^{\dagger} U_{A} \sigma_{w} U_{A}^{\dagger}$. Furthermore, it can be verified using the commutation and anticommutation laws for Pauli matrices, that $\sigma_{v}$ and $\sigma_{w}$ are commutable operators if and only if $v^{T} P w=0$. Since $\sigma_{v}$ and $\sigma_{w}$ are commutable if and only if $U_{A} \sigma_{v} U_{A}^{\dagger}$ and $U_{A} \sigma_{w} U_{A}^{\dagger}$ are commutable, it must hold that $v^{T} A^{T} P A w=v^{T} P w$ for all $v$ and $w$, which proves $A^{T} P A=P$.

To prove part (ii), we will first consider $n=2$ and show that all permutations $\pi: x \rightarrow A x$ with $A^{T} P A=P$ can be generated with the operations $\phi_{u}: \tilde{\Psi} \rightarrow U_{u} \tilde{\Psi} U_{u}^{\dagger}$ with $U_{u}=e^{i \frac{\pi}{4} \sigma_{u}}=\frac{1}{\sqrt{2}}\left(I+i \sigma_{u}\right.$ ) (with $\left.u \in \mathbb{Z}_{2}^{4}\right)$. (This is also true for $n>2$, but generators affecting more than 2 qubits at a time will not be needed). Using $\sigma_{v} \sigma_{w}=$ $(-1)^{v^{T} P w} \sigma_{w} \sigma_{v}$, it can be shown that $\phi_{u}$ translated into the binary language results in a permutation $\pi_{u}: x \rightarrow$ $x+u\left(u^{T} P x\right)=\left(I+u u^{T} P\right) x$.

We will now show that the group of permutations generated by the permutations $\pi_{u}$ is isomorphic to $S_{6}$, the group of all permutations of 6 elements. Next we will show that the group of $P$-orthogonal $4 \times 4$ matrices contains $6 !=720$ elements, which proves that all $P$-orthogonal permutations are generated. Since no permutation (except the identity) is commutable with all permutations, $S_{6}$ is isomorphic to the group of transformations $\chi_{q}: S_{6} \rightarrow S_{6}: p \rightarrow q p q^{-1}$ where $p$ and $q$ are permutations of 6 elements. Such a transformation $\chi_{q}$ is completely determined by specifying the images of the 15 commutations $p_{i, j}$, permutations on $\{1,2,3,4,5,6\}$ that permute $i$ and $j$. This holds because any permutation is a composition of such commutations and $\chi_{q}\left(p_{1} p_{2}\right)=\chi_{q}\left(p_{1}\right) \chi_{q}\left(p_{2}\right)$. Note that the image under $\chi_{q}$ of a commutation is again a commutation. As a result $S_{6}$ is isomorphic to the group of permutations of 15 elements obtained by restricting $\chi_{q}$ to the commutations. We will show that this is exactly the group of permutations generated by the generators $\pi_{u}$ (which can be considered as permutations of 15 elements as 0000 can be left out, being always mapped to itself). To this end we establish the following correspondence between nonzero 4-bit vectors and commutations $\gamma: u \rightarrow p_{i, j}$ :

$$
\begin{aligned}
& 0001 \rightarrow p_{5,6}, \quad 0010 \rightarrow p_{4,6}, \quad 0011 \rightarrow p_{4,5}, \\
& 0100 \rightarrow p_{2,3}, \quad 0101 \rightarrow p_{1,4}, \quad 0110 \rightarrow p_{1,5}, \quad 0111 \rightarrow p_{1,6}, \\
& 1000 \rightarrow p_{1,3}, \quad 1001 \rightarrow p_{2,4}, \quad 1010 \rightarrow p_{2,5}, \quad 1011 \rightarrow p_{2,6}, \\
& 1100 \rightarrow p_{1,2}, \quad 1101 \rightarrow p_{3,4}, \quad 1110 \rightarrow p_{3,5}, \quad 1111 \rightarrow p_{3,6} .
\end{aligned}
$$

It can be verified that $\gamma\left(\pi_{u}(x)\right)=\chi_{\gamma(u)}(\gamma(x))$ for all $u$ and $x$. So $\pi_{u}$ and $\chi_{\gamma(u)}$ realize the same permutation of 
15 elements. As a consequence, also products $\pi_{u_{1}} \cdot \ldots \cdot \pi_{u_{k}}$ realize the same permutations as products $\chi_{\gamma\left(u_{1}\right)} \cdot \ldots$. $\chi_{\gamma\left(u_{k}\right)}$. This finally establishes the isomorphism between $S_{6}$ and the permutations generated by the $\pi_{u}$.

It remains to be shown that there are $6 ! P$-orthogonal $4 \times 4$ matrices. It follows from $A^{T} P A=P$ that $A$ is $P$-orthogonal if and only if all pairs of columns of $A$ represent commutable $\sigma_{a_{i}}$ except for the first and second or the third and fourth column. Therefore to make an arbitrary $P$-orthogonal matrix, the first column $a_{1}$ can be chosen to be any nonzero 4 -bit vector (15 choices), the second column should satisfy $a_{1}^{T} P a_{2}=1$ (one linear condition yielding 8 possible $a_{2}$ ), the third column should be commutable with $a_{1}$ and $a_{2}$ (two linear conditions yielding 3 choices after excluding 0000) and finally the fourth column should be commutable with $a_{1}$ and $a_{2}$ and noncommutable with $a_{3}$ (three linear conditions, yielding 2 possibilities). This results in $15 \cdot 8 \cdot 3 \cdot 2=720=6$ ! possibilities. This ends the proof for $n=2$.

For $n>2$ we turn to the matrix picture and show that every $P$-orthogonal matrix $A$ can be reduced to the identity matrix by two-qubit operations, i.e. $4 \times 4 P$ orthogonal matrices embedded in an identity matrix on rows and columns $2 k+1,2 k+2,2 l+1,2 l+2$ for some $k, l \in\{0, \ldots, n-1\}$. We concentrate on two columns of $A$ at a time, first 1 and 2 , then 3 and 4 and so on and transform them to the corresponding columns of the identity matrix with two-qubit operations. Assume, without loss of generality, that we are working on columns 1 and 2 , then we name $K^{(k, l)}=A_{\{2 k+1,2 k+2,2 l+1,2 l+2\},\{1,2\}}$. If the two columns of $K^{(k, l)}$ are commutable they can be thought of as the first and third column of a $4 \times 4 P$ orthogonal matrix and can be reduced by a two-qubit operation to the first and third column of an identity matrix. If the two columns of $K^{(k, l)}$ are noncommutable they can be reduced to the first and second column of an identity matrix. One can see that by combining such two qubit operations the first two columns of $A$ can be reduced to the first two columns of an identity matrix. Due to the commutability relations between the columns of $A$, as a result, also the first two rows become the first rows of an identity matrix. One can now proceed in a similar way with the next pairs of columns until the whole matrix is reduced to the identity matrix. The composition of the inverses of all two-qubit operations that were applied yields a decomposition of $A$ into two-qubit operations that can be realized by local unitary operations as shown above. This ends the proof.

In the proof we saw that linear transformations $(b=0)$ correspond to operations with $U_{B}=U_{A}^{*}$, i.e. $\tilde{\Psi} \rightarrow$ $U_{A} \tilde{\Psi} U_{A}^{\dagger}$. The matrices $U_{A}$ that under this action map tensor products of Pauli matrices to tensor products of Pauli matrices possibly with a minus-sign are known to form the Clifford group, studied in [7, 8] in the context of quantum error correction and quantum computation. The P-orthogonal matrices form a group that is isomorphic to a quotient group of the Clifford group. The Clifford group is known to be generated by CNOT opera- tions and one-qubit operations that map Pauli matrices to Pauli matrices. It is possible that this knowledge may be used to give other proofs for the theorem above. However, we think that our set of generators and the isomorphism between $P$-orthogonal $4 \times 4$ matrices and permutations of 6 elements are worthwhile results in their own. It also follows that the CNOT operation should be decomposable in terms of our generators (at least up to phase factors, but we can do better). One can easily verify that $\mathrm{CNOT}=\frac{1+i}{\sqrt{2}} e^{-i \frac{\pi}{4} \sigma_{1000}} e^{i \frac{\pi}{4} \sigma_{1001}} e^{-i \frac{\pi}{4} \sigma_{0001}}$. Note that the first and last operation are actually 1-qubit operations.

\section{MIXED STATE MULTI-COPY ENTANGLEMENT DISTILLATION FROM PAIRS OF QUBITS}

The distillation protocols presented in this paper can be summarized as follows.

1. Start from $n$ identical independent Bell diagonal states with entanglement. This yields a mixture of $4^{n}$ tensor products of Bell states.

2. Apply a local permutation of these $4^{n}$ products of Bell states as described in the previous section. As a result the $n$ qubit pairs get statistically dependent.

3. Check whether the last $n-m$ qubit pairs are $|\Phi\rangle$ states $\left(\left|\Phi^{+}\right\rangle\right.$or $\left.\left|\Phi^{-}\right\rangle\right)$. This can be accomplished locally by measuring both qubits of each pair in the $|0\rangle,|1\rangle$ basis, and checking whether both measurements yield the same result.

4. If all measured pairs were $|\Phi\rangle$-states, keep the first $m$ pairs. This is a new mixture of $4^{m}$ products of Bell states.

This is a generalization of a protocol with $n=2$ and $m=1$, presented in [2, 3. In that protocol the applied local permutation consisted of a bilateral CNOT operation by Alice and Bob. In our protocol, we will only consider linear permutations $(b=0)$ as one can easily see nothing can be gained by considering affine permutations. In the next section we discuss how to choose the local permutation so as to obtain a good protocol. The main result of this section is a formula for the resulting state of $m$ pairs as a function of the permutation of Bell states performed in step 2 of the protocol:

Theorem 2 If Alice and Bob apply the above protocol, starting from $n$ independent identical copies of a Bell diagonal state $p_{00}\left|\Phi^{+}\right\rangle\left\langle\Phi^{+}\left|+p_{01}\right| \Psi^{+}\right\rangle\left\langle\Psi^{+}\left|+p_{10}\right| \Phi^{-}\right\rangle\left\langle\Phi^{-}\right|+$ $p_{11}\left|\Psi^{-}\right\rangle\left\langle\Psi^{-}\right|$with $p_{00} \geq p_{01} \geq p_{10} \geq p_{11}$, and with entanglement, i.e. $p_{00}>\frac{1}{2}$, with in step 2 a local permutation, $\pi: x \rightarrow A x$ with $A^{T} P A=P$, the resulting state of the remaining $m$ qubit pairs is given by

$$
2^{n-m} \sum_{y \in \mathbb{Z}_{2}^{2 m}}\left(\frac{\sum_{x \in \mathcal{S}+P A^{T} P \bar{y}} p_{x}}{\sum_{x \in \mathcal{S}} s_{x}}\right)\left|B_{y}\right\rangle\left\langle B_{y}\right|
$$


where $\mathcal{S}$ is the subspace spanned by the rows of $A P$ with indices $2 m+2,2 m+4, \ldots, 2 n$,

$$
\left[\begin{array}{l}
s_{00} \\
s_{01} \\
s_{10} \\
s_{11}
\end{array}\right]=\left[\begin{array}{rrrr}
1 & 1 & 1 & 1 \\
1 & 1 & -1 & -1 \\
1 & -1 & 1 & -1 \\
1 & -1 & -1 & 1
\end{array}\right]\left[\begin{array}{l}
p_{00} \\
p_{01} \\
p_{10} \\
p_{11}
\end{array}\right],
$$

$\bar{y}$ is y extended with $2(n-m)$ zeros, and the long vector indices of $p$ and $s$ and $B$ behave like the indices of $\sigma$ in the previous section, e.g. $p_{001101}=p_{00} p_{11} p_{01}$.

Proof: After the permutation, and before the measurement, the state of the $n$ qubit pairs is given by $\sum_{x \in \mathbb{Z}_{2}^{2 n}} p_{x}\left|B_{A x}\right\rangle\left\langle B_{A x}\right|$. The states $\left|B_{A x}\right\rangle$ with $(A x)_{2 m+2},(A x)_{2 m+4}, \ldots,(A x)_{2 n}=0$ yield $|\Phi\rangle$-states and will be kept. These are the states $\left|B_{A x}\right\rangle$ for which $x$ is commutable with the rows $a_{2 m+2}, \ldots, a_{2 n}$ of $A P$. If we call the subspace of these vectors $x, \mathcal{R}$, the success rate (probability of keeping the first $m$ pairs) is $\sum_{x \in \mathcal{R}} p_{x}$. Among the states that are kept, the ones with $(A x)_{j}=y_{j}, j=1, \ldots, 2 m$ yield $\left|B_{y}\right\rangle$-states. Together with the conditions for being kept, these are $2 m+(n-m)$ independent linear conditions, yielding a coset of an $(n-m)$-dimensional subspace of $\mathbb{Z}_{2}^{2 n}$. This subspace must be $\mathcal{S}$ since the latter is $(n-m)$-dimensional and satisfies all homogeneous conditions (with $y=0$ ) by the $P$-orthogonality of $A$. The right coset is obtained by adding $P A^{T} P \bar{y}$ (a combination of the first $2 m$ rows of $A P$, determined by $y$ ). As a result the state of the first $m$ pairs after the measurement is

$$
\sum_{y \in \mathbb{Z}_{2}^{2 m}}\left(\frac{\sum_{x \in \mathcal{S}+P A^{T} P \bar{y}} p_{x}}{\sum_{x \in \mathcal{R}} p_{x}}\right)\left|B_{y}\right\rangle\left\langle B_{y}\right|
$$

If all coefficients (for all $y$ ) are calculated, the denominator $\sum_{x \in \mathcal{R}} p_{x}$ can be calculated as the sum of the $2^{2 m}$ numerators. If only one coefficient is needed (for instance if only the fidelity of the end state is needed) the denominator can be calculated in a more efficient way as $\sum_{x \in \mathcal{R}} p_{x}=2^{-(n-m)} \sum_{x \in \mathcal{S}} s_{x}$. One can easily verify that $s_{v}=\sum_{x \in \mathbb{Z}_{2}^{2 n}}(-1)^{v^{T} P x} p_{x}$ (First verify for 2 bits and then extend). Therefore $\sum_{v \in \mathcal{S}} s_{v}=$ $\sum_{x \in \mathbb{Z}_{2}^{2 n}}\left(\sum_{v \in \mathcal{S}}(-1)^{v^{T} P x}\right) p_{x}$. If $x$ commutes with all $v \in$ $\mathcal{S},(-1)^{v^{T} P x}=1$ for all $v \in \mathcal{S}$ and $\sum_{v \in \mathcal{S}}(-1)^{v^{T} P x}=$ $2^{n-m}$. If $v \notin \mathcal{S}$, one can easily show that half the coefficients $(-1)^{v^{T} P x}$ are 1 and half are -1 . Now the states $x \in$ $\mathcal{R}$ are exactly the ones for which $x$ is commutable with all elements of $\mathcal{S}$. Therefore $\sum_{x \in \mathcal{S}} s_{x}=2^{n-m} \sum_{x \in \mathcal{R}} p_{x}$. This concludes the proof.

\section{RECURRENCE SCHEMES}

With the above formula for the end state of the protocol (Theorem 2), it is possible to derive good protocols by searching over all possible values for the relevant rows of the $P$-orthogonal matrix $A$ and optimizing some quality measure. Typically this measure will depend on the fidelity of the end state and the success rate of the protocol (the probability of having $|\Phi\rangle$-states in the measured pairs). In that case, one only needs the first coefficient (the fidelity) and the denominator (the success rate) in (5), which both only depend on $\mathcal{S}$, a space spanned by only $n-m$ rows of $\mathcal{S}$. Although this drastically limits the search space, it still grows exponentially with growing $n$.

Therefore, to come up with schemes for large $n$, one needs to use the recurrence scheme, as was proposed for $n=2$ and $m=1$ in [2, 3]. If $m=1$, this scheme means that the above protocol is performed $n$ times (with the same local permutation) and the $n$ identical end states are taken as the input for a new step. Of course more than two steps are possible too. One could also envision recurrence schemes with $m \neq 1$, for instance, combining two end states of an $n=4, m=2$-protocol to yield the input for a second step with $n=4$. In that case however the input for the second step would no longer consist of $n$ independent pairs. Although this only requires a minor modification of the above results $\left(p_{x}\right.$ and $s_{x}$ can no longer be interpreted as products of $\left.p_{00}, \ldots, p_{11}\right)$, we will not consider this case in this paper.

To end up with almost pure Bell states, the recurrence scheme can best be combined with the hashing protocol as in [2, 3. The hashing protocol is the best known asymptotic protocol (for $n \rightarrow \infty$ ) but can only be applied to Bell diagonal states with high enough fidelity. The combined protocol then consists of first applying a few recurrence steps and then switching to the hashing protocol.

The best known $n=2, m=1$-recurrence scheme is the one of [6]. In our language it amounts to a scheme with a $4 \times 4 P$-orthogonal matrix whose last line is 1111 . It can be proven that this scheme yields the best achievable fidelity after one step (though not achieved with the best success rate) for initial probabilities that are ordered $p_{00}>p_{01} \geq p_{10} \geq p_{11}$. For this reason it is also best to apply a pair per pair transformation after each recurrence step, which reorders the probabilities of the end state if they are not ordered. (One can easily find such one-pair transformations using the theory of section II or equivalently using the local operations of [2, 37.)

Although it is probably best to search for a new protocol for every given initial state, we propose below a protocol which we think is good if one does not have the time for such a search. We show by computer simulations that it performs better than the $n=2$ scheme.

Our scheme is an $n=4, m=1$ recurrence scheme combined with hashing, and with as the last step possibly an $n=2, m=1$-step if this can lead to better performance. For the local permutation (determined by the $P$-orthogonal matrix $A$ ) we choose a permutation that is found experimentally to often lead to the best fidelity after one step, when starting with ordered probabilities. For this reason we also apply a 


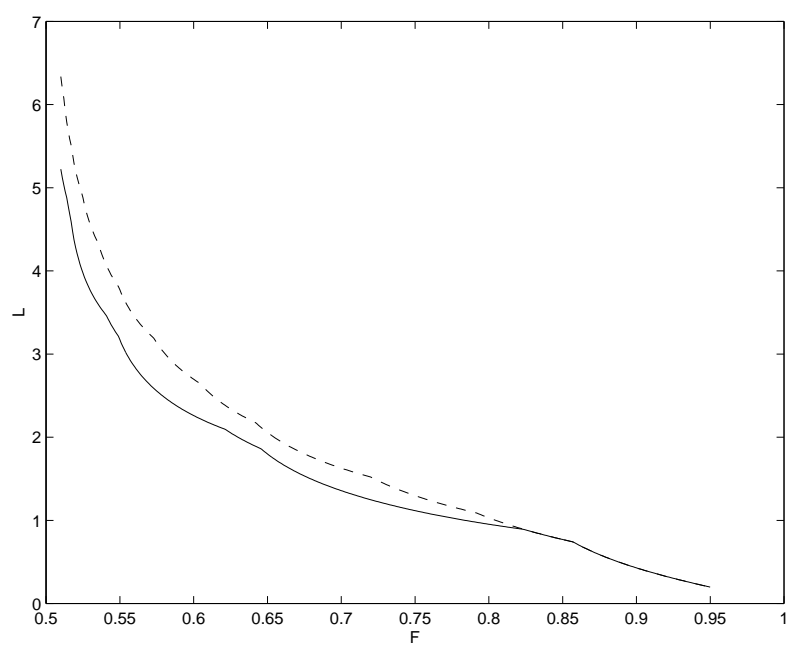

FIG. 1: Comparison of 10-logarithm of inverse asymptotic yield $\mathrm{L}$ for input Werner states with fidelity $\mathrm{F}$ for proposed protocol (full line) and existing recurrence/hashing protocol (dashed line)

reordering in between recurrence steps as discussed for the $n=2$-protocol above. The chosen local permutation corresponds to an $8 \times 8 P$-orthogonal matrix $A$ whose fourth, sixth and eighth row span the space spanned by $\{10111110,01101100,11101011\}$. This can be achieved by the operations

$$
\begin{aligned}
U_{A}=U_{B}^{*}= & e^{i \pi / 4 \sigma_{10010000}} e^{i \pi / 4 \sigma_{01000001}} \\
& e^{i \pi / 4 \sigma_{10001100}} e^{i \pi / 4 \sigma_{00011000}} .
\end{aligned}
$$

In this realization the first and second row of the $P$ orthogonal matrix $A$ are 01100010 and 10101010 . These rows are needed to compute the reordering operations between the steps, for although the three values of $p_{01}^{\prime}, p_{10}^{\prime}$ and $p_{11}^{\prime}$ after one step of the protocol are fixed, their order is not. (The three cosets of $\mathcal{S}$ in $\mathcal{R}$ in equation (5) are fixed but not their order).

This realization was found by exhaustive search over all operations that can be realized by 4 consecutive elementary two-qubit operations. If, for protocols with larger $n$ for instance, no such simple realization can be found in a reasonable amount of time, one can always find a realization using the theory of section II but this can increase the total amount of work for the distillation protocol. This was also one of the reasons for choosing $n=4$ in the proposed protocol.

As a performance measure we have chosen the expected average number of input pairs needed per output Bell state in an asymptotic protocol (the inverse of the asymptotic yield). The number of recurrence steps was also chosen as to optimize this measure. Fig. 1 shows the performance for our method $(n=4, m=1$ recurrence with the local permutation realized by $U_{A}$ as in (6), with reordering between the steps, possibly one last $n=2, m=1$-step, and optimal switching to hashing protocol) and the method of [6] with reordering between the steps and optimal switching to the Hashing protocol. For the sake of simplicity the figure only shows the results for Werner states (with $p_{00}=F>\frac{1}{2}$ and $\left.p_{01}=p_{10}=p_{11}=\frac{1-F}{3}\right)$, but the method also performs better for non-Werner states.

To do better than this protocol for a specific initial Bell diagonal state, one can do several things depending on the amount of computing time available. One can try recurrence schemes with higher $n$ and even higher $m$, but the amount of time needed increases fast with increasing $n$. There is of course no obligation to take the same local permutation in consecutive recurrence steps. One can also consider distilling more than one end state at once. Making two states with two $n=4, m=1$-protocols is just a special case of a non-optimal $n=8, m=2$ protocol. One can of course search for better ones if one has the time. In this case, the two obtained Bell states will not be independent but as the fidelity goes to 1 , their dependence will vanish. Also two consecutive recurrence steps, say two $n=2, m=1$-steps, can be considered as one bigger non-optimal step, in this case with $n=4, m=1$. So if one has the time one can in theory always go for a one shot protocol (no recurrence), but if one combines with the recurrence scheme one can always afford lower initial entanglement with the same amount of computing time.

\section{CONCLUSION}

We have derived new protocols for distillation of entanglement from mixed states of two qubits. The protocols were based on a characterization of the group of all locally realizable permutations of the $4^{n}$ possible tensor products of $n$ Bell states. Our protocols perform significantly better than existing protocols as was shown by computer simulation. We also indicated how to derive even better protocols for specific initial states.

\section{Acknowledgments}

Our research is supported by grants from several funding agencies and sources: Research Council KULeuven: Concerted Research Action GOA-Mefisto 666 (Mathematical Engineering), several $\mathrm{PhD} /$ postdoc \& fellow grants; Flemish Government: Fund for Scientific Research Flanders (several $\mathrm{PhD} /$ postdoc grants, projects G.0256.97 (subspace), G.0240.99 (multilinear algebra), research communities ICCoS, ANMMM)); Belgian Federal Government: DWTC (IUAP IV-02 (1996-2001) and IUAP V-22 (2002-2006): Dynamical Systems and Control: Computation, Identification \& Modelling); The European Commission: Esprit project: DICTAM 
[1] F. Verstraete, J. Dehaene, and B. D. Moor, Physical Review A 64, 010101 (2001).

[2] C. Bennett, D. DiVincenzo, J. Smolin, and W. Wootters, Phys. Rev. A 54, 3824 (1996).

[3] C. Bennett, G. Brassard, S. Popescu, B. Schumacher, J. Smolin, and W. Wootters, Phys. Rev. Lett. 76, 722 (1996).

[4] M. Horodecki, Quantum Information and Computation 1, 3 (2001).

[5] E. Rains, IEEE transactions on Information Theory 47,
2921 (2001).

[6] D. Deutsch, A. Ekert, R. Josze, C. Macchiavello, S. Popescu, and A. Sanpera, Phys. Rev. Lett. 77, 2818 (1996)

[7] D. Gottesman, The Heisenberg representation of quantum computers, quant-ph/9807006.

[8] D. Gottesman, Ph.D. thesis, Caltech (1997), quantph/9705052. 